\title{
PIC based Vehicular Monitoring and Tracking System with Android Application
}

\author{
Anjali B, S Uma
}

\begin{abstract}
The proposed configuration depends on Vehicular tracking and monitoring utilizing PIC microcontroller. The vehicular module is utilized to track, monitor, and surveillance and finds the accident spot and personal to the observing station. The proposed outline gives data with respect to vehicle Identity, speed, and position on ongoing premise. This data are gathered by the PIC and SMS is received by android application. The android application gets the receiver data and displays the parameters. The vehicle tracking module introduced inside of the vehicle sends an SMS containing the GPS directions to the client, utilizing which he tracks the vehicle on Google Earth. The user could certainly forwards the SMS, consisting the GPS coordinates to his dear companions and relatives on the off chance that he wishes to, with the goal that they can likewise track the vehicle utilizing Google Earth.
\end{abstract}

Keywords: PIC, GPS, GSM, Wireless monitoring station, Vehicle monitoring, Vehicle tracking.

\section{Introduction}

In today's reality as the population builds step by step the quantities of vehicles additionally increments on the road and highways. This outcome in more accidents that leads to traffic jam. This module gives data about the accident to the hospital and a nearby police station. Accordingly sudden level of supervision and administration of freight transport vehicles, particularly trucks conveying coal it is vital to create transport vehicles remote monitoring module [2]. A server PC at the (remote) monitoring station that is persistently waiting for information from the system, need to record the activities of the vehicle into a database. This contains the data with respect to Vehicle speed,position, identity and temperature in two designs. The data given to monitoring station is in persistent way and when the accidents happen. The advancement of vehicular outline gets open numerous accommodation life additionally brings numerous issues in the meantime, for instance, traffic congestion, trouble in observing dispersive vehicle, robbery and other problems[4].

\section{Vehicle Tracking System \& Monitoring System}

Vehicle Tracking System (VTS) is the generation used to determine the region of a vehicle the use of one of kind techniques like GPS and different radio navigation structures running thru satellites and floor based total stations. Via following triangulation or trilateration strategies the monitoring device allows to calculate easy and correct location of the car. Automobile data like area details, velocity and distance travelled and so on. It can be considered on a virtual map with the help of software through the net. Even records may be stored and downloaded to a computer from the GPS unit at a base station and that could later be used for analysis. This device is an important tool for tracking each automobile at a given time period and now it is becoming increasingly more popular for human beings having steeply-priced automobiles and hence as a theft prevention and retrieval device.

The system includes modern hardware and software additives allowing one to track their vehicle online or offline. Any automobile monitoring gadget consists of specifically three components mobile automobile unit, fixed based totally station and, database and software program gadget.

1) Vehicle Unit: it is the hardware factor connected to the vehicle having both a GPS/GSM modem. The unit is configured around a number one modem that capability with the tracking software program by way of receiving signals from GPS satellites or radio station factors with the help of an antenna. The controller modem converts the information and sends the car place records to the server.

2) Fixed Based Station: includes a wireless community to receive and ahead the information to the statistics Centre. Base stations are prepared with tracking software and geographic map useful for determining the car area. Maps of each metropolis and landmarks are to be held in the based station that has an inbuilt internet Server.

3) Database and software program: the placement records or the coordinates of each journeying point are stored in a database, which later may be regarded in a display using digital maps. However, the users must connect them to the internet server with the respective vehicle identification saved in the database and best then can view the region of vehicle travelled.

Vehicle monitoring system describes digital device this is used to tune, check and report on vehicle interest which in turn reflects on the vehicle conduct. Whether it's far a complicated telematics gadget this is suited for the car itself, a GPS, or simply a smart telephone app, the objectives are the identical:

1) To reduce incidents on the road.

2) Deliver efficiency in road transport operations.

Increasingly, vehicle manufacturers are fitting new vehicles with this technology as standard and in some countries, it is becoming mandatory. It's also known as telematics or onboard units, are available for both private motorists and for companies with a fleet of vehicles. When used privately, it is often in the context of identifying areas for improvement for inexperienced younger drivers and insurers offer reduced premiums to drivers who use such systems. 


\section{The Hardware System}

\section{GSM Modem Section}

This phase consists of a GSM modem. The modem will communicate with microcontroller the use of serial conversation. The modem is interfaced to microcontroller the use of MAX 232, a serial driver.

EEPROM: This section acts as a backend database for the mission. This phase is found out using an EEPROM incorporated circuit chip.

THERMISTOR: Thermistors are a temperature sensing device. It's far used to sense the temperature. In this project through relying upon on the value of temperature the exhaust fan will run.

GPS MODEM: A GPS modem is used to get the signals and receive the alerts from the satellites. In this task, GPS modem gets the alerts from the satellites and people are given to the microcontroller. The indicators may be within the form of the coordinates; these are represented in the shape of the latitudes, longitudes and altitudes. Function (longitude, latitude) identification and temperature to the monitoring station and to the user/owners cellular that ought to help them to get scientific assist if an accident or the robbery. We're supposed to make this tracking Wi-Fi using a microcontroller. In today's global accidental deaths are growing every day in all most all accidents the loss of life triggered due to loss of remedies in time, due to no longer getting facts to the nearest hospitals and police station right now because of the existing present method of calling any character to the clinic and police station.

GSM MODEM: The GSM modem is a specialized type of modem which accepts a SIM card operates on a subscriber's mobile number over a network, much like a mobile phone. It is a mobile phone without a show. Modem $\operatorname{sim} 300$ is a triband GSM/GPRS engine that works on EGSM900MHz, DCS1800MHz and PCS1900MHz frequencies. GSM Modem is RS232-common sense stage well matched, i.e., it takes- $3 \mathrm{v}$ to $-15 \mathrm{v}$ as good judgment high and $+3 \mathrm{v}$ to +15 as a logic low.MAX232 is used to convert TTL into RS232 good judgment degree converter used among the microcontroller and the GSM board. The sign at pin eleven of the microcontroller is dispatched to the GSM modem via pin 11 of max232.This signal is received at pin2 (RX) of the GSM modem. The GSM modem transmits the signal from pin3 (TX) to the microcontroller thru MAX232, that's obtained at pin 10 of IC1.

SHOCK SENSOR: Pressure sensor is used to convert pressure as physical Quantity into electric impulses. The stress sensor used right here is piezoelectric sensor.

GAS SENSOR: A gas detector is a device that detects the presence of gases in a place, frequently as part of a protection gadget. This sort of system is used to detect on a gasoline leak and interface with a manipulate gadget so a system may be mechanically shut down. A gas detector can sound an alarm to operators within the area wherein the leak is occurring, giving them the opportunity to go away. This kind of device is vital due to the fact there are many gases that may be harmful to natural life, including human beings or animals.

\section{PIC 16f877a}

PIC 16f877a is the name for the Microchip microcontroller (MCU) own family, which includes a microprocessor, I/O ports, timers and other internal, incorporated hardware. The principle benefits are low outside component rely, a wide range of chip sizes terrific availability of compilers and source code and easy programming. These $\mu \mathrm{c}$ 's are available with a number capabilities packaged with each twin in-line (DIP) applications and surface-mount applications. These are available in 28 pin DIP, 40 pin DIP, 44 pin surface mount package, etc.

\section{Block Diagram of Proposed Tracking System}

The block diagram of tracking system using GPS and GSM technology is presented in figure 1. The proposed system is vehicle tracking and navigation system where the location of the vehicleis traced with the help ofPIC 16f877a microcontroller, GPS receiver, GSM modem. GPSand GSM pins Transceiver and Receiver pins are connected to PIC controller serial ports. The serial communication of data takes place. At the beginning, it takes the data from the GPS receiver and then sends the information in the form of SMS with the help of GSM modem to the user. The baud rate of the GPS receiver is 9600 . At the time of processing, GPS is receiving GPRMC values.

The microcontroller obtains latitude and longitude values obviating time, Satellite name, User authentication, etc. GSMis a Global system for mobile communication device which works as an SMS Sender and Receiver. The power supplied to the components and Micro control circuitry using a $12 \mathrm{~V}$ or battery. GSM requires $12 \mathrm{v}$, GPS and PIC requires $5 \mathrm{v}$.

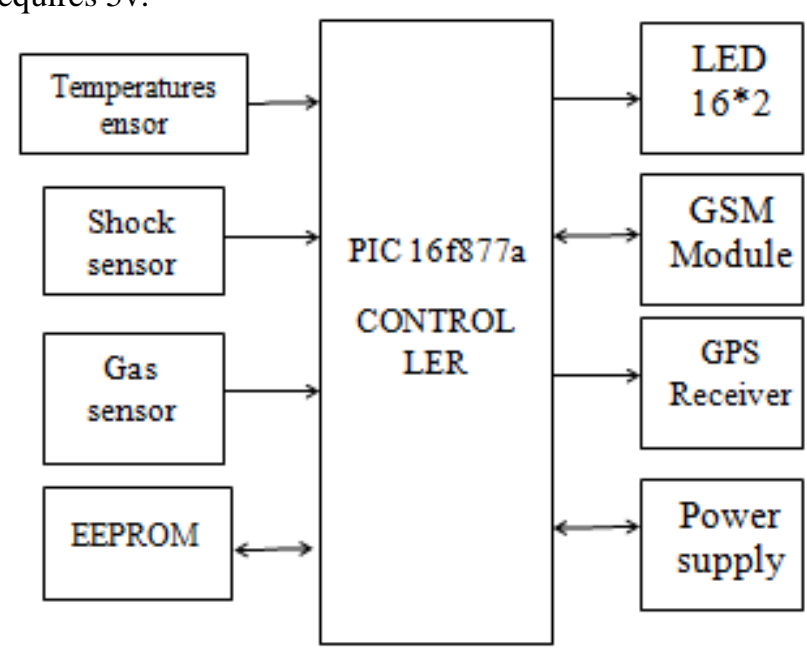

Figure 1: Block Diagram of Proposed System

\section{Overview Development of the Android}

Android is a Linux-based OS for mobile devices such as smartphones and tablet computers [3]. In proposed android application is used to access the sensor information like vehicle Identity location, velocity, location, temperature, gas detector. The Android mobile within the vehicle receives the

\section{Volume 5 Issue 6, June 2016} www.ijsr.net 


\section{International Journal of Science and Research (IJSR) \\ ISSN (Online): 2319-7064}

Index Copernicus Value (2013): 6.14 | Impact Factor (2015): 6.391

latitudinal information from the satellite through GPS and the GPRS enabled SIM sends message. The user can track the vehicle on a Google map.

\section{Development of the Android Application Software}

Our Android mobile application software developed on a Windows platform for an Android phone [1] [3]. The environment preparation software which included the Java development kit (JDK), Eclipse, Android software development kit (SDK), Android virtual devices (AVD). Meanwhile, Android development tools (ADT) is the plug-in through which Eclipse is customized for Android application development. It provides a powerful, integrated environment and draws out the functions of Eclipse that lets users create applications rapidly and add components on the API. AVD is an accumulation of virtual devices where each AVD simulates a virtual device to extend the Android platform and test the application software before they are run (tested) on the actual physical mobile phone (device).

\section{Tracking flow chart}

The application starts first select the position of the vehicle and send the data to the satellite and then find the vehicle using the GPS coordinates sent by the satellite, after verifying if they found vehicle is that of the owner. If the vehicle is that of the owner we stop otherwise we add the data to the target set which is then sent to the satellite as shown in figure 2 .

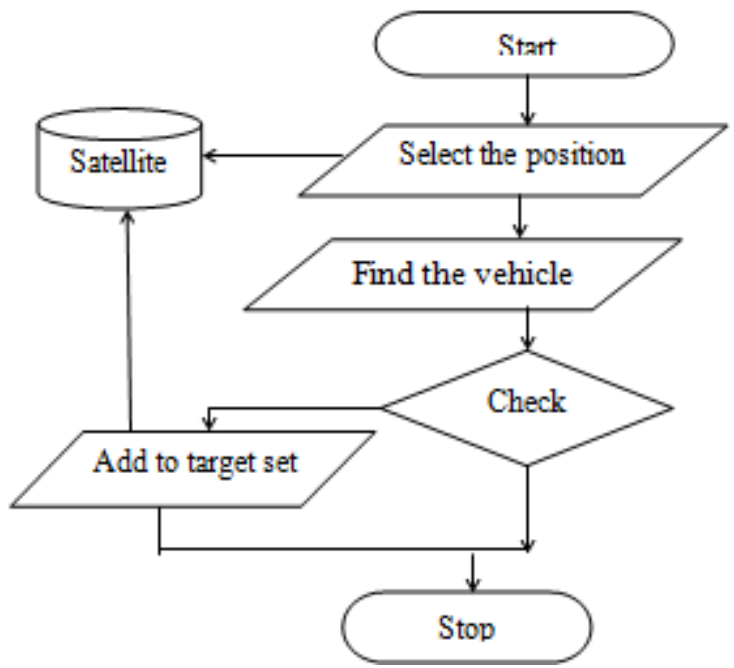

Figure 2: Flow Chart of Tracking System

\section{SMS Receiver Application on Android Phone}

i) The application is activated on message received from the tracking system.

ii) It traces the location of the SMS message from the system.

iii) It exposesthe current position of the user.

iv) SMS receiver uses Internet connection and automatically seeks the location on Google Map.

v) It is very user friendly and easy to understand.

vi) Received SMS Containing Latitude-Longitude on Smartphone

vii) It guides to the current location of the user by Google map. At the receiving end and the output of GMAP is obtained.
The proposed model was tested on different subjects at different position. When a subject or leaves the zone defined or call is made or received by the GSM modem it sends the coordinates received by the GPS module to user in SMS (short message services) [5] [8].

\section{Conclusion}

The Vehicular System provides information of a vehicle like velocity, position, through a GPS module and identity of a vehicle to a monitoring station and to a mobile phone according to a definite event stored in a program or a query from a monitoring station. The accelerometer senses the collision of the vehicle and sends this information in real time to a hospital/police station.

The monitoring station display this information on Android application. The system is useful in many applications such as surveillance, security, tracking, which may be installed in cargo trucks, cars, motorcycle, and boat to retrieve location and vehicle status information and send it to the other stationary module then the receiving module which collects the transmitted information by SMS and process it in a compatible format to Google Earth to view the location and vehicle status online.

\section{References}

[1] Angel, Gonzalez Villan, Joseph JorbaEsteve, "Remote Control of Mobile Devices in Android Platform". IEEE, 2013.

[2] Hui Hu, Lian Fang, published a paper title "Design and Implementation of Vehicle Monitoring System Based on GPS/GSM/GIS", Third International Symposium on Intelligent Information technology Application.2009.

[3] C. Haseman, Android Essentials, Berkeley, CA: A Press, 2008.

[4] Lu Xutao1, Cui DongSen2 "Design of Transport Vehicles Remote Monitoring System", 2nd International Conference on Education Technology and Computer (ICETC). 2010.

[5] M.P.Patelguda, Ibrahimpatnam, Ranga Reddy, published a paper on title "Vehicle Tracking and Identifying Based on Android", on International Journal of Engineering Trends and Technology Oct 2014.

[6] Peng Chen, "ShuangLiu, Intelligent Vehicle Monitoring System Based on GPS, GSM and GIS", WASE International Conference on Information Engineering. 2010.

[7] Rousan, A. R. AI-Ali and K. Darwish, published a paper title" GSM-Based Mobile Tele Monitoring and Management System for Inter- Cities Public Transportations", International Conference on Industrial Technology (ICIT). 2004 IEEE.

[8] SayaliNerkar, Shweta Jadhav, on title "Android Based Vehicle Service Status Monitoring System"on International Journal of Advanced Research in Electrical, Electronics and Instrumentation Engineering, February 2014.

[9] Zhang Wen, Jiang Meng "Design of Vehicle positioning System Based on ARM", Business Management and Electronic Information (BMEI), International Conference 2011 IEEE.

Volume 5 Issue 6, June 2016 www.ijsr.net 